\title{
Sensing Capacitance of Underwater Objects in Bio-inspired Electrosense
}

\author{
Yang Bai ${ }^{1}$, James Snyder ${ }^{2}$, Yonatan Silverman ${ }^{1}$, Michael Peshkin ${ }^{1}$ and Malcolm A. MacIver ${ }^{1,2,3}$
}

\begin{abstract}
Certain electric fish use a self-generated AC electric field to navigate and hunt. Thousands of sensors on the surface of the fish's body detect the pattern of amplitude and phase distortions of the field caused by nearby objects. Prior research has suggested that phase distortions may be especially useful for recognition of live objects. Here we present the first study of the utility of phase information in a robotic implementation of active electrosense. Using our robotic implementation, we investigated how the phase information depends on the frequency of the emitted field, the conductivity of the surrounding water, and object properties. An analytical model was developed serving as qualitative explanation of the dependency. We show that in certain situations phase information enables discrimination between two objects that are otherwise very similar in the amplitude of their electric images. We also show the utility of probing objects with multiple frequencies.
\end{abstract}

\section{INTRODUCTION}

Certain species of fish, in two distinct geographical regions (South America and Africa) [12] have evolved the capacity to emit a weak AC electric field and detect distortions of that field for navigation and hunting of prey. This unusual sensory modality is termed "active electrosense." Active electrosense has been extensively studied in biology [20] as a model system for understanding how sensory signals are processed by the nervous system. Studies have shown that weakly electric fish have separate neural pathways for the phase and amplitude components of electric images [15], [21], [3], [5]. Recently, robotic analogs of active electrosense have been created [17], [18], [1] for use in underwater robot sensing applications as well as for gaining insight into mechanisms of biological electrosense. These prior efforts have focused on obtaining and analyzing the amplitude component of the pattern of field distortions by the sensors scattered over the body surface (the "electric image"). In this study, we focus on the extraction and analysis of the phase component of electric images. A block diagram

This work was supported by NSF grants IOB-0517683 and CMMI-0941674 to M.A.M., and an Office of Naval Research Small Business Technology Transfer grant N00014-09-M-0306 to M.P. and M.A.M.

${ }^{1}$ Department of Mechanical Engineering, R.R. McCormick School of Engineering and Applied Science, Northwestern University, Evanston, Illinois, United States of America

${ }^{2}$ Department of Biomedical Engineering, R.R. McCormick School of Engineering and Applied Science, Northwestern University, Evanston, Illinois, United States of America

${ }^{3}$ Department of Neurobiology, Northwestern University, Evanston, Illinois, United States of America of our mobile implementation (hereafter "sensorPod") is illustrated in Fig. 1. One electrode emits an AC voltage while the other electrode is grounded. Sensors along the sides are in differential pairs (marked black) and are used to pick up voltage signals. The essence of active electrosense is to detect minute variations on top of a large signal. In the case of real fish, sensitivity falls in the range of 1:1000-1:10,000 (1.0-0.1 $\mu \mathrm{V}$ on an emitted signal of $\approx 1 \mathrm{mV}$ ) [13], [6]. In our implementation, by subtracting the readings from a geometrically symmetric sensor pair, the common mode signal is canceled, leaving only a small perturbation to be amplified. By demodulating the amplified differential signal with a reference signal from the same frequency source, a sensitive measure of signal amplitude is extracted. Tasks such as wall following and simple object detection can be accomplished using signal amplitude variations [17], [18], [8]. However, if the amplified signal is also demodulated with a reference signal that is $\pi / 2$ out of phase, both phase and magnitude can be obtained. Such phase extraction is the basis of the current study.

\section{PRINCIPLE}

\section{A. Dual Channel Demodulation}

Our electronics creates an extra phase shift, mostly due to the high pass filter (Fig. 1). We remove this phase shift with a rotation matrix. If the differential signal to detect is $A \sin (\omega t+\phi)$, the two demodulated channel readings $V_{\mathrm{ch} 1}, V_{\mathrm{ch} 2}$ are,

$$
\begin{aligned}
& V_{\mathrm{ch} 1}=\text { Gain } \cdot A \sin (\phi) \\
& V_{\mathrm{ch} 2}=\text { Gain } \cdot A \cos (\phi)
\end{aligned}
$$

Using trigonometric operations and known Gain, the amplitude $A$ and phase $\phi$ are separated. In the figures below, "Ch1" and "Ch2" represent these dual channel demodulated and rotated readings.

\section{B. Object Fly-by Profile}

Sensory data from two channels yield explicit information about the electrosense signal's magnitude and phase, encoding the complex impedance of the environment. The impedance depends on a variety of factors including the water's dielectric properties, the dielectric properties and geometry of objects in the water, and the excitation frequency. Under simplified ideal conditions of a medium with uniform conductivity $\sigma_{1}$ and permittivity $\epsilon_{1}$, uniform electric field $\mathbf{E}_{\mathbf{0}}$, an analytical solution for the voltage perturbation due to a spherical object of uniform material properties $\sigma_{2}$ and $\epsilon_{2}$ has been derived [14]. Using 


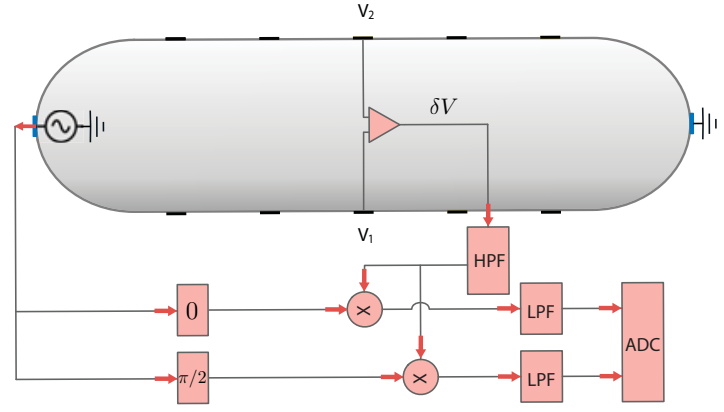

Fig. 1. SensorPod configuration and demodulation circuit. The differential reading from the middle sensor pair is demodulated by the same source used for excitation.

a polar coordinate system based on the object $(\mathbf{r}, \theta)$ as illustrated in Fig. 2, this is expressed as

$$
\delta \Phi(d)=E_{0} \sin ^{2} \theta \cos \theta\left(\frac{a^{3}}{d^{2}}\right) \frac{\rho_{1}-\rho_{2}+i \omega \rho_{1} \rho_{2}\left(\epsilon_{1}-\epsilon_{2}\right)}{\rho_{1}+2 \rho_{2}+i \omega \rho_{1} \rho_{2}\left(2 \epsilon_{1}+\epsilon_{2}\right)}
$$

Here $d$ is the distance of the object ( $d_{0}$ in Fig. 2 ) from the sensorPod and $\rho$ is resistivity, the inverse of conductivity $\sigma$. As the sensorPod passes by the object to be sensed, the electrosense reading presents a profile, which we refer to as the "fly-by profile," as shown in Fig. 2. This profile with a zero-crossing and peak is observable in both data channels and is not restricted to the ideal case. The fly-by profiles for insulating and conducting objects are different [18]. The profiles are broadly opposites of each other: insulators and conductors with identical geometries will have profiles of opposite polarity and differing magnitude. The phase of the induced perturbation should be 0 for conductive objects and $\pi$ for insulating objects.

\section{Object Phase Spectrum}

For capacitive objects, water conductivity and the frequency of the electric field probe are major factors affecting phase. Conductivity influences phase in a relative manner. For natural capacitive objects, like aquatic plants, the less conductive the water is the easier the object is to detect. For tap water at around $300 \mu \mathrm{S} / \mathrm{cm}$, aquatic plants appear to be insulating while in water of $40 \mu \mathrm{S} / \mathrm{cm}$ (typical of many South American rivers where electric fish live), aquatic plants are capacitive (for Apteronotus fish with an emission frequency of around $1 \mathrm{kHz}$.) Probe frequency is also crucial. Frequency is easy for us to manipulate over a wide range with a programmable waveform generator and wide-band circuitry. In the biological case, emitted signals typically contain multiple frequency components [19], [2], although it is unclear whether the fish exploits these components for electrolocation.

\section{IMPLEMENTATION}

\section{A. Hardware Setup}

Our experimental platform includes an X-Y robot mounted over a tank [18]. The emitting electrodes on the

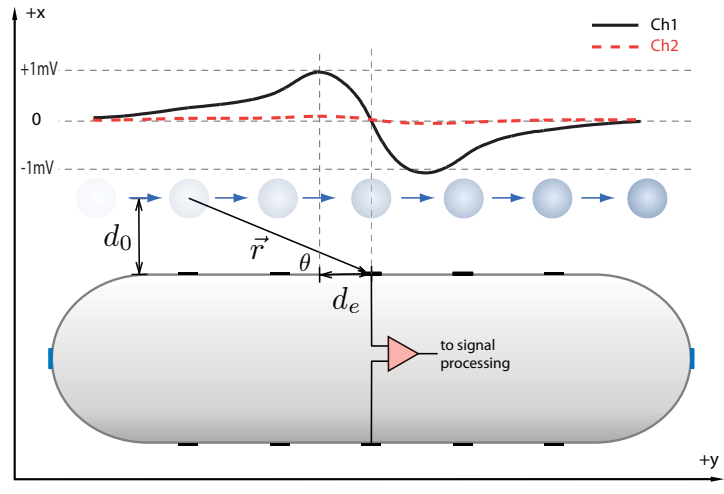

Fig. 2. A dual channel (solid black and dashed red) fly-by profile of an object moving in the $+\mathrm{y}$ direction. The black and red traces show the demodulated and rotated signal resulting from a plastic object flying by.

sensorPod were excited by a waveform generator at $4 \mathrm{~V}$ peak to peak amplitude with tunable frequency. Only the middle pair of electrodes were used as detectors. The demodulation circuitry is shown schematically in Fig. 1. Targets used in experiments include manufactured capacitors and resistors as well as real-world objects for which only qualitative electrical properties are known. Capacitors and resistors were mounted on an insulating holder and placed in the water. The leads of the electrical components were connected to electrodes at the bottom of the holder. We subtracted the effect of the insulating holder from the electrical images we obtained for the components in the holder. When we studied real-world objects, such as metal, plastic cubes, and grapes, they were attached by a skewer or string that had minimal electric image impact and were easy to subtract.

\section{B. Fly-by Experiments}

Fly-by Operation. Targets were moved by the sensorPod at a fixed velocity and at a fixed distance. Each experiment was run twice, once for the holder or skewer alone and once with the target object for subtraction. For example, Fig. 2 shows the fly-by operation with the sensorPod shown as reference. Because of the insulating nature of the object (plastic), there is no phase shift (except for a sign flip of $\pi$ ). The sign flip happens when the object crosses the zero reading point in the middle but is not shown in Fig. 2. One channel exhibits a sinusoidal-like profile while the other channel is nearly featureless. In wall following tasks, only one channel is used and the relative amplitude is used as the cue to maintaining a desired distance from the wall.

Capacitor/Resistor Fly-by. Fig. 3 shows the two channels' readings of a $22 \mathrm{nF}$ and $1 \mu \mathrm{F}$ capacitor in our holder at a bath conductivity of $350 \mu \mathrm{S} / \mathrm{cm}$, typical of tap water. The qualitative difference in phase is easily observed. We measured the phase reading for manufactured capacitor and resistor values of $22 \mathrm{nF}, 50 \mathrm{nF}, 100 \mathrm{nF}, 1 \mu \mathrm{F}, 100 \Omega$ 


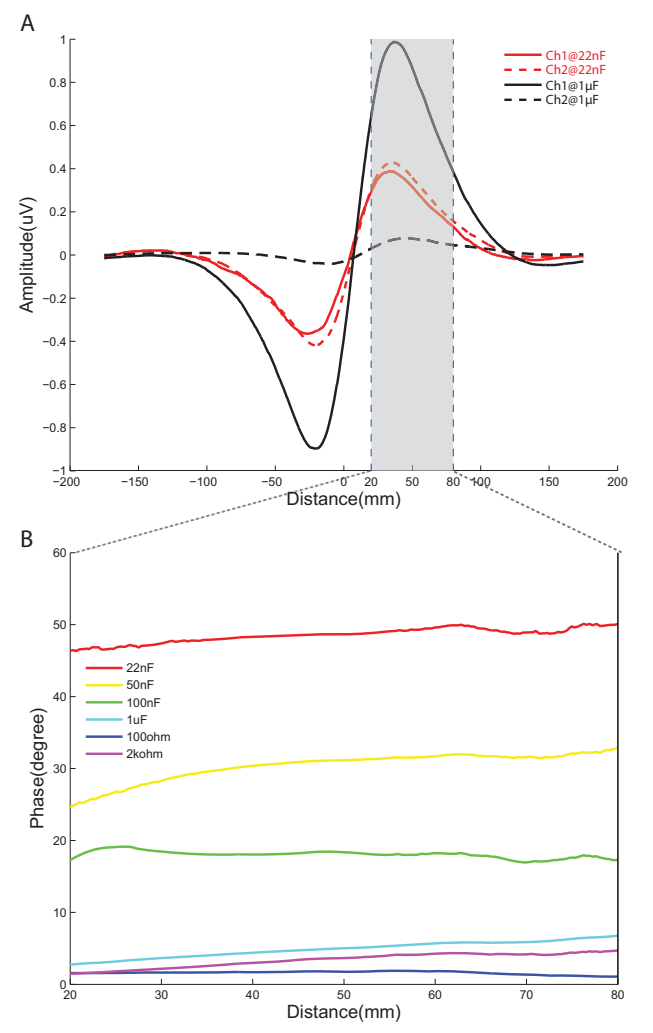

Fig. 3. (A) Dual channel (solid black and dashed red) fly-by profiles of $22 \mathrm{nF}$ and $1 \mu \mathrm{F}$ capacitors. (B) Phase profile of $22 \mathrm{nF}, 50 \mathrm{nF}$, $100 \mathrm{nF}$, and $1 \mu \mathrm{F}$ capacitor, as well as $100 \Omega$ and $2 \mathrm{k} \Omega$ resistors over the range of $20-80 \mathrm{~mm}$ (shaded area in (A)).

and $2 \mathrm{k} \Omega$. All components were scanned from -180 to $180 \mathrm{~mm}$ full range $(-80$ to $80 \mathrm{~mm}$ is shown in Fig. 3) and phase reading from within the 20 to $80 \mathrm{~mm}$ range is shown. For the trials in Fig. 3, the 20 to $80 \mathrm{~mm}$ range is where the readings are larger, making them more easily interpreted. For both capacitors and resistors, the phase stayed roughly the same over this range. As capacitance increases, the phase decreases and approaches 0 due to lowered impedance. For resistors, the phase exhibited no change and remained around 0 as expected.

Conductivity and Frequency. The influence of conductivity and frequency was studied using a capacitor of known value. Fig. 4 shows the fly-by profiles of a $22 \mathrm{nF}$ capacitor under varying bath conductivities and varying frequency conditions. Increased frequency changes the phase of the electric image of the capacitor toward the ideal conductor (zero phase) situation. The decrease of water conductivity also brings the phase closer to the ideal conductor condition. The reason is that for a less conducting environment, the complex impedance of the capacitor appears to be more conductive. As these measurements indicate, conductivity and frequency are two global parameters that influence the phase of a capacitive object in water and have qualitatively similar effects.
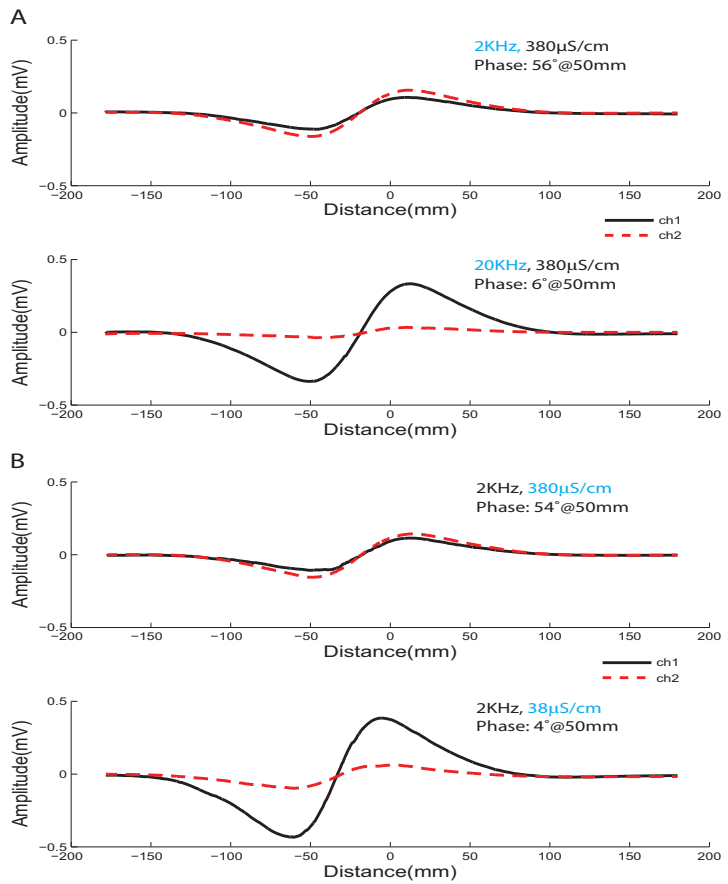

Fig. 4. Dual channel (solid black and dashed red) fly-by profiles of a $22 \mathrm{nF}$ capacitor at $(\mathrm{A})$ different frequencies $(2 \mathrm{kHz}$ and $20 \mathrm{kHz})$ and (B) different conductivities $(380 \mu \mathrm{S} / \mathrm{cm}$ and $38 \mu \mathrm{S} / \mathrm{cm})$. The phase is interpreted with the holder at $50 \mathrm{~mm}$. Note that zero-crossings should occur at the center of the sensorPod. The different zerocrossings in the figures are due to different experimental setups.

Real Object Fly-by. Cubes of plastic, metal (steel), and fresh meat (lamb) were scanned to show the profiles of objects with diverse properties, shown in Fig. 5. The choice of the objects was based on their nature and convenience. The metal cube used was not anodized but it quickly formed a water-metal interface when submerged [11]. This interface contributes capacitance, making the phase of metal diverge from the ideal conductor case. The fresh lamb gave close to 0 phase similar to a purely conductive object [16]. If compared with the profile of the plastic cube in Fig. 2, the profile of a purely conductive object is flipped in sign. For a conductive object, one of the channels gives negative readings and then positive readings after it crosses the zero reading point. In the process, the other channel remains at zero. Fig. 6 shows that a grape $(\approx 2.5 \mathrm{~cm}$ in diameter $)$ is a capacitive object. Its profile changes considerably when the bath conductivity is varied. As noted, frequency and bath conductivity have the same effect in terms of altering phase, so it is desirable to vary frequency to study capacitive objects.

Rock vs Aquatic Plant. Here we characterize the electric images of a rock versus a semi-aquatic plant, the ribbon plant (Dracaena sanderiana) because these two objects are commonly found in aquatic environments. The bath conductivity was $40 \mu \mathrm{S} / \mathrm{cm}$ in order to mimic the fresh water environment typical of the native habitat of South American electric fish [10]. Fig. 7 shows that 

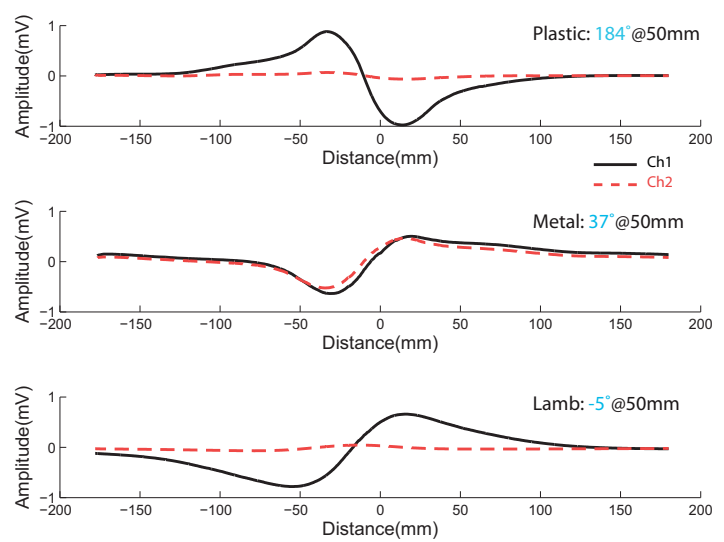

Fig. 5. Dual fly-by profiles (solid black and dashed red) of plastic, metal, and lamb cubes at $2 \mathrm{kHz}, 380 \mu \mathrm{S} / \mathrm{cm}$. The phase is interpreted with the samples at $50 \mathrm{~mm}$.
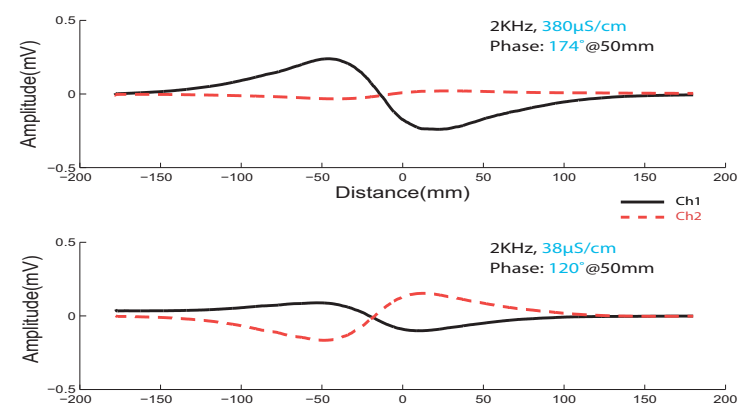

Fig. 6. Dual channel (solid black and dashed red) fly-by profiles of grape at different conductivity conditions $(380 \mu \mathrm{S} / \mathrm{cm}$ and $38 \mu \mathrm{S} / \mathrm{cm}$ ). The phase is interpreted with the grape at $50 \mathrm{~mm}$.

both profiles have one channel that give very similar readings in shape and amplitude, while the other channel is quite different for the two objects. This experiment demonstrates the utility of using two out-of-phase channels to perform real world electrolocation tasks.

\section{Probing With Multiple Frequencies}

Operation. To study the frequency response of signals caused by real objects, we used excitation frequencies of $2 \mathrm{kHz}, 5 \mathrm{kHz}, 10 \mathrm{kHz}, 20 \mathrm{kHz}, 50 \mathrm{kHz}$, and $100 \mathrm{kHz}$. Because grapes are known to be capacitive (due to their membrane) and are easy to mount on a skewer, they were selected as the target object to study frequency, size and distance influence on phase, see Fig. 8.

Probe Frequency. The influence of excitation frequency on phase is evident with spectrum analysis. As shown in Fig. 8 , the phase component of a grape's electric image exhibits significant change over the frequency range we used. At low frequency, the grape appeared to be insulating with close to $\pi$ phase. As the frequency increases, the phase decreases and crosses $\frac{\pi}{2}$ and the signature approaches a perfect conductor. Unlike bath conductivity, frequency is easily adjustable. Thus, fre-
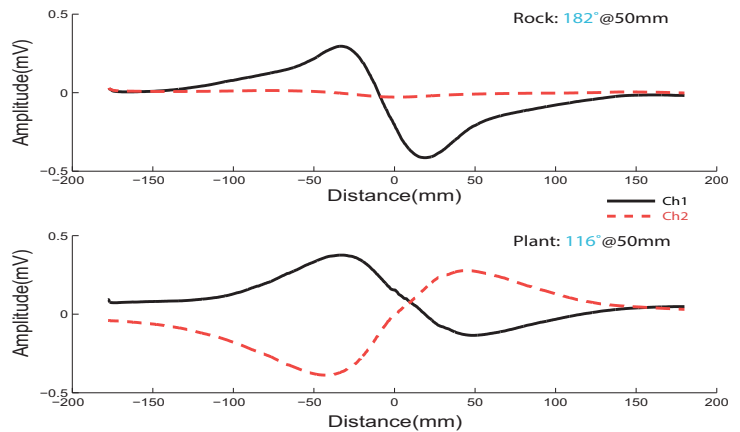

Fig. 7. Dual channel (solid black and dashed red) fly-by profiles of rock and aquatic plant in $40 \mu \mathrm{S} / \mathrm{cm}$ low conductivity water. The phase is interpreted with the objects at $50 \mathrm{~mm}$.
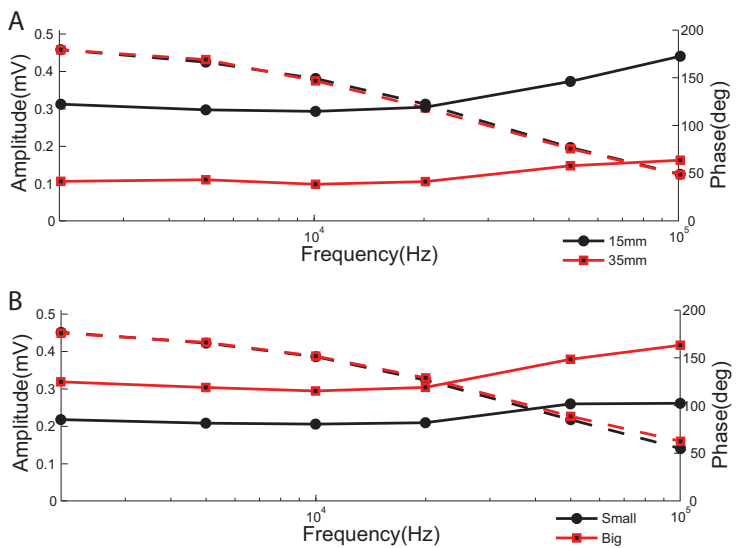

Fig. 8. Phase and amplitude spectrum of grapes of (A) different distances $(15 \mathrm{~mm}$ and $35 \mathrm{~mm})$ and (B) different sizes $(50 \%$ variation). For both objects, phase curves are dotted.

quency scanning is a practical approach to extracting certain properties of objects within the sensory range of electrolocation systems.

Target Distance. A grape was placed at distances of $15 \mathrm{~mm}$ and $35 \mathrm{~mm}$ perpendicular to the long axis of the sensorPod. The resulting phase and amplitude is shown in Fig. 8A. Distance has no effect on the phase information for this particular object. In future work, we will examine how broadly the invariance of phase with distance holds across different kinds of objects. In contrast, the influence of distance on the magnitude of the electric image is very pronounced. For objects at the two measured distances $(15 \mathrm{~mm}$ and $35 \mathrm{~mm}$ ), the magnitude profiles increase in inverse proportion to distance.

Target Size Two grapes varying in size by $50 \%$ were placed at the same location while the frequency was varied. As shown in Fig. 8B, despite this size variation there was very little change in phase over the frequency range.

Nylon Net vs. Aquatic Plant. A one-layer nylon screen was rolled into a bundle close to the size of the plant. 


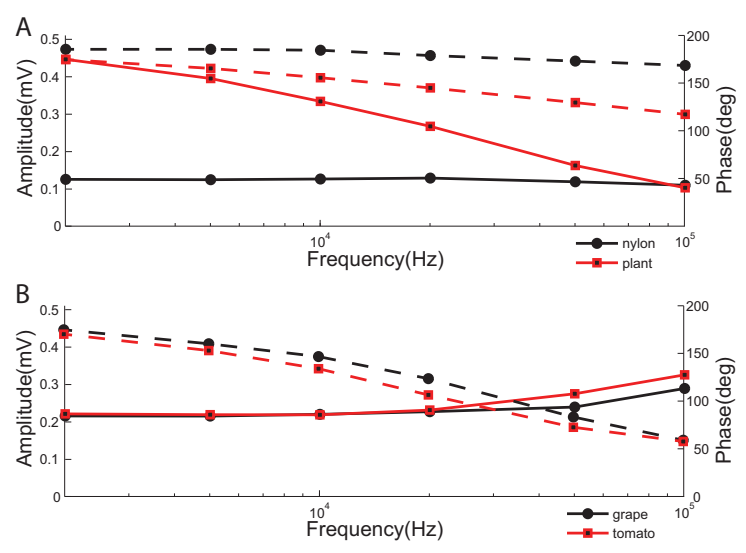

Fig. 9. Phase and amplitude spectrum comparison (A) between nylon net and aquatic plant and (B) between grape and cherry tomato of the same size (both $\approx 2.5 \mathrm{~cm}$ in diameter). For both objects, phase curves are dotted.

Its size and that of the aquatic plant were larger than all the previously tested objects. They extended from the surface of the water to the depth of the sensors (Fig. 9A). The purpose of this experiment was to motivate the use of information available only with two channels. For the nylon net, both phase and magnitude remain relatively constant over frequency. For the plant, at low frequency it appears to more like an insulator, similar to the nylon net; however, its capacitive properties are revealed as the probe frequency increases. Both phase and amplitude decrease with the increase of excitation frequency for the plant, uniquely differentiating it from the nylon net.

Grape vs Cherry Tomato. Grapes and cherry tomatoes of approximately identical geometry were tested to obtain their signal spectrums, see Fig. 9B. These two objects are very similar in appearance. However, the phase spectrum shows a discernible difference which reflects the difference of in dielectric properties.

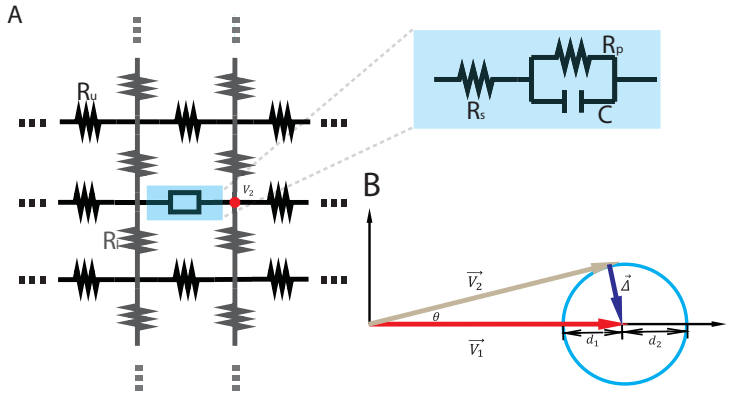

Fig. 10. (A) Part of the lumped element resistor network model of water body and object made up of unit resistor $R_{u}$ and link resistor $R_{l}$ (B) vector graph of frequency influence on signal magnitude and phase. $\vec{V}_{1}$ is reading from the unperturbed side with fixed phase and magnitude, $\overrightarrow{V_{2}}$ is reading from the perturbed side with varying phase and magnitude. $\vec{\Delta}$ is the differential signal, $\vec{\Delta}=\overrightarrow{V_{1}}-\overrightarrow{V_{2}}$.

\section{Discussion}

\section{A. Analytical Model}

Previous studies [4] have used quasi finite element simulation with lumped elements to study the electric image of objects with complex impedance. [4] used a 2D model and studied the case of electric fish on a crosssection.For our robotic implementation, the field can also be studied computationally using finite element methods. However, a simple model that explains phase is desirable as well. Therefore, rather than solving Maxwell's equations with boundary conditions [7], we took an intuitive approach by focusing on the electric signal path and developed a lumped element model. The purpose of this model is to qualitatively explain phase dependency on geometric and electrical parameters. We assume that an object's distance, size, and shape are weak influencing factors of phase. First, an object is modeled as a certain configuration of lumped resistors and capacitors. Second, the water body is modeled as a resistor, the value of which can be measured directly. For $400 \mu \mathrm{S} / \mathrm{cm}$ water, the resistance seen by the sensorPod's two excitation electrodes was measured to be on the $1 \mathrm{k} \Omega$ level. So the water body is modeled as a cylindrical sheet (not a crosssection as in [4]) of connected resistors that wrap around the sensorPod. The resistor network is made up of unit resistor $R_{u}$ and link resistor $R_{l}$ as shown in Fig. 10A. The introduction of the object in the water was effectively replacing a block of water with the object's equivalent model (the case shown in Fig. 10). When the object's complex impedance is not equal to that of the water replaced, there is current going through the link resistor $R_{l}$. Fig. 10B gives a vector explanation of the phase and magnitude spectrum of different objects. $\vec{V}_{1}$ refers to the signal on the side of the sensorPod with no perturbation. It is of zero phase and fixed length. $\vec{V}_{2}$ is the signal that changes with frequency. For the case of conductive or insulating objects, $\theta$ is always 0 . For a capacitive object at different frequencies, its angle increases from 0 to a maximum and then decreases back to 0 . The difference $\vec{\Delta}$ is what is demodulated and analyzed. If the object's complex impedance eventually goes smaller than that of the water it is replacing, then $\vec{\Delta}$ experiences a $\pi$ phase change from $\pi$ to 0 . For small perturbation, $\theta$ is very small. So with approximation, the frequency at which the angle of $\vec{\Delta}$ is $\pi / 2$ is also when the object's complex impedance is the same as unit resistor $R_{u}$. This give a critical frequency $\omega_{\pi / 2}$. The magnitude $\Delta$ also changes with frequency. As has been seen in Fig. 8 and Fig. 9, the magnitudes at 0 and $\pi\left(d_{1}, d_{2}\right.$ respectively) are not necessarily the same. The magnitude either decreases or increases as frequency increases. As a lumped electrical element, this object should therefore be a resistor in series with a capacitor and another resistor in parallel, Fig. 10A. Inequalities of those values can be derived based on phase variation. $\theta$ reaches its maximum value $\theta_{\max }$ at frequency $\omega_{\max }$. This frequency makes $\vec{V}_{2}$ tan- 
gential to $\vec{\Delta}$. When the angle variation is small, the comparison between $d_{1}$ and $d_{2}$ determines the location of $\omega_{\max }$. When $d_{1}$ is smaller than $d_{2}, \omega_{\max }$ is smaller than $\omega_{\pi / 2}$. In reality, the single-ended signal's phase variation is extremely hard to measure due to noise, which is another reason why we chose differential mode detection. Using this model in a circuit simulation tool (SPICE) with appropriate values of resistors and capacitors, the phase and amplitude profiles of objects were qualitatively similar to measurements.

\section{B. Application}

Capacitive sensing gives significantly more information than single channel sensing. Fig. 7 shows a simple fresh water environment navigation task. With only one channel it would be far harder to discriminate a rock from an aquatic plant.

Object discrimination could be performed with phase sensing and the lumped element model. Differences between capacitive objects and conductive/insulating objects are easily seen from bode plots. For two capacitive objects with similar shapes, more discriminating power can be found within the framework of the model by comparing $\omega_{\pi / 2}, d_{1,2}$ and other features such as slopes. In general, object identification includes the retrieval of an object's dielectric strength, its 3D geometry, and distance. It is an inverse problem that is difficult to solve and almost always ill-posed [9]. Our preliminary studies with finite element approach suggest that at very high frequency, the object's phase and amplitude are only dependent on its geometry. This indicates that an object's identification could be separated into two tasks, geometry extraction and electrical property extraction. Phase sensing serves as the starting point of a bottom-up approach to general object identification with artificial electrosense.

\section{CONCLUSIONS}

This study concerns the novel and important capacitive sensing component of artificial electrosense. Capacitive sensing, by using an additional demodulation channel, presents additional information that is difficult or impossible to attain from a single channel. Capacitance was shown to affect phase, conditioned on water conductivity and operating frequency. Phase information encodes aspects of the ambient fluid conductivity when considered across multiple frequencies, but is also influenced by target distance and object geometry. Within the background of real-world applications, the usefulness of capacitive sensing was shown. This study also serves as the foundation of the more general object identification problem using artificial electrosense.

\section{REFERENCES}

[1] F. Boyer, P. B. Gossiaux, B. Jawad, V. Lebastard, and M. Porez. Model for a sensor inspired by electric fish. Robotics, IEEE Transactions on, 28:492-505, 2012.
[2] A. A. Caputi, M. E. Castello, P. Aguilera, and O. TrujilloCenoz. Electrolocation and electrocommunication in pulse gymnotids: signal carriers, pre-receptor mechanisms and the electrosensory mosaic. Journal of Physiology-Paris, 96(56):493-505, 2002. Caputi, AA Castello, ME Aguilera, P Trujillo-Cenoz, O.

[3] CE. Carr. Neuroehology of electric fish. BioScience, 40:259$267,2011$.

[4] K. Fujita and Y. Kashimori. Modeling the electric image produced by objects with complex impedance in weakly electric fish. Biol Cybern, 103:105-118, 2010.

[5] H. G and T. H. Bullock. The detection of electric fields from electric organs. In A. Fessard, editor, Electroreceptors and other specialized receptors in lower vertebrates (Handbook of sensory physiology, vol III/3), pages 201-256. Springer, 1974.

[6] J B M Goense and R Ratnam. Continuous detection of weak sensory signals in afferent spike trains: the role of anti-correlated interspike intervals in detection performance. Journal of comparative physiology. A, Neuroethology, sensory, neural, and behavioral physiology, 189(10):741-59, October 2003.

[7] J. D. Jackson. Classical Electrodynamics. John Wiley \& Sons, New York, NY, 1962.

[8] V. Lebastard, C. Chevallereau, A. Amrouche, B. Jawad, A. Girin, F. Boyer, and P.B. Gossiaux. Underwater robot navigation around a sphere using electrolocation sense and kalman filter. 2010 IEEE/RSJ International Conference on Intelligent Robots and Systems, pages 4225-4230, 2010.

[9] William Lionheart, Nicholas Polydorides, and Andrea Borsic. The reconstruction problem. In Electrical Impedance Tomography, Series in Medical Physics and Biomedical Engineering. Taylor \& Francis, December 2004.

[10] M. A. MacIver, N. M. Sharabash, and M. E. Nelson. Preycapture behavior in gymnotid electric fish: Motion analysis and effects of water conductivity. J. Exp. Biol., 204(3):543$557,2001$.

[11] E.T. McAdams, A Lackermeier, J.A. McLaughlin, D Macken, and J. Jossinet. The linear and non-linear electrical properties of the electrode-electrolyte interface. Biosensors and Bioelectronics, 10(1-2):67-74, January 1995.

[12] P. Moller. Electric Fishes: History and Behavior. Chapman \& Hall, London, 1995.

[13] M. E. Nelson and M. A. MacIver. Prey capture in the weakly electric fish Apteronotus albifrons: Sensory acquisition strategies and electrosensory consequences. J. Exp. Biol., 202(10):1195-1203, 1999.

[14] B. Rasnow. The effects of simple objects on the electric field of Apteronotus. J. Comp. Physiol. A, 178(3):397-411, 1996.

[15] B. Rasnow and J. M. Bower. The electric organ discharges of the gymnotiform fishes: I. Apteronotus leptorhynchus. J. Comp. Physiol. A, 178(3):383-396, 1996.

[16] S. Sarang, S. Sastry, and L. Knipe. Electrical conductivity of fruits and meats during ohmic heating. Journal of Food Engineering, 87:352-356, 2008.

[17] James R. Solberg, Kevin M. Lynch, and Malcolm A. MacIver. Robotic electrolocation: Active underwater target localization with electric fields. In International Conference on Robotics and Automation, Rome, Italy, April 10-14, 2007. IEEE.

[18] James R. Solberg, Kevin M. Lynch, and Malcolm A. MacIver. Active electrolocation for underwater target localization. International Journal of Robotics Research, 27(5):529-548, 2008.

[19] C. R. Turner, M. Derylo, C. D. de Santana, J. A. AlvesGomes, and G. T. Smith. Phylogenetic comparative analysis of electric communication signals in ghost knifefishes (gymnotiformes : Apteronotidae). Journal of Experimental Biology, 210(23):4104-4122, 2007. Turner, Cameron R. Derylo, Maksymilian de Santana, C. David Alves-Gomes, Jose A. Smith, G. Troy.

[20] R. W. Turner, L. Maler, and M. Burrows. Special issue on electroreception and electrocommunication. J. Exp. Biol., 202(10):1167-1458, 1999.

[21] G. von der Emde. Capacitance detection in the wave-type electric fish eigenmannia during active electrolocation. $J$. Comp. Physiol. A, 182(2):217-224, 1998. 